(REVIEW ARTICLE)

\title{
Community pharmacists: Frontline COVID-19 warriors and the approaches for the safe management of the pharmacies during the pandemic
}

\author{
Desai Deepa Mahendra ${ }^{1,}{ }^{*}$, Patil Rupali Bhushan ${ }^{2}$ and Desai Yash Mahendra ${ }^{3}$ \\ ${ }^{1}$ Department of Pharmaceutical Chemistry, N.S.S. College of Pharmacy, Maharashtra State Board of Technical Education, \\ Mumbai, India. \\ 2 Department of Pharmacognosy, N.S.S. College of Pharmacy, Maharashtra State Board of Technical Education, Mumbai, \\ India. \\ ${ }^{3}$ Department of General Medicine, Geetanjali Medical College and Hospital, Udaipur, India.
}

Publication history: Received on 08 May 2020; revised on 22 May 2020; accepted on 24 May 2020

Article DOI: https://doi.org/10.30574/wjarr.2020.6.2.0150

\begin{abstract}
In today's scary scenario of the outbreak of Corona Virus Disease (COVID-19) pandemic and nation-wide lockdown, most of the business houses including majority of public services have come to a stand-still. But in this devastating condition, there is a dire need of health care professionals and health care services. In such times, apart from the Doctors and Nurses, the role of Community pharmacist has become very significant and crucial. This article focusses on some of the guiding principles for the community pharmacists for the safe running of their pharmacies during the pandemic.
\end{abstract}

Keywords: COVID-19; Pandemic; Lockdown; Community Pharmacists

\section{Introduction}

Today the severe acute respiratory syndrome coronavirus 2, known as COVID-19 is the current health distress to the whole world. How much ever is the readiness of any government with different disaster management plans, this was completely beyond imagination and beyond the reach of any such plans. None of the countries for sure had given any formal training to the people or the healthcare professionals to combat such a disaster.

In this scary scenario of the out-break of Corona Virus Disease (COVID-19) pandemic and nation-wide lockdown, most of the businesses including majority of public services have come to a halt, as the government has declared the business of only the essential goods and services to be operational. In such times, there is a dire need of health care professionals and health care services, where apart from the Doctors and Nurses, the role of Community Pharmacists has become crucial and important. Community pharmacies are probably the first point of contact, in both the outbreak affected and non-affected areas. Ensuring the continuity of their services to the population is very important [1].

As always but particularly at this time, community pharmacies are on the frontline in the health care service. There is a tremendous increase in the demand for medicines, particularly the analgesics and antipyretics along with an increased demand for the life-saving medicines due to panic buying [2],[3]. Highly increased demand for medicines was seen in the initial few days of the lockdown 1.0, due to the fact that the announcement of the lockdown 1.0 was quite sudden, not giving much time to the patients to procure their stock beforehand. Although it was announced that the pharmacies will remain open, there was a panic buying of the medicines at least for a month or even more.

During the pandemic, community pharmacies are helping vulnerable patients stay well by providing the supply of medicines and their operations can make a big difference in this critical time. Obviously the possibility of exposure to COVID-19 of these frontline pharmacists does exist as they have to interact with the patients who may possibly be

\footnotetext{
${ }^{*}$ Corresponding author: Desai Deepa Mahendra
} 
infected. Hence there has to be proper guidelines as to what are the best practices which community pharmacies should implement amidst this pandemic to ensure the safety of the patients/community at large and of the staff at the pharmacy.

\section{Some of the important facets to the precautionary approaches are:}

\subsection{Strategize to minimize the physical contact between the staff and the customers and also between the customers}

Numerous innovative preventive measures can be implemented rapidly as a part of such strategy:

\subsubsection{Using Engineering controls}

Based on the experience of the countries which got affected by the COVID-19 pandemic much before us, such as China, America and Italy and on the guidance from eminent epidemiologists and physicians, the government, from time to time, issues guidelines indicating the preventive measures to be adopted during the outbreak of this virus. One of the most important guidelines on which every government is stressing is Social Distancing [4]. The guidelines advise that all individuals maintain a distance of at least 1 meter $/ 6$ feet apart to protect oneself from getting infected with COVID-19 virus infection from a person already infected [5].

Community Pharmacies should devise ways and techniques to enforce this for patients and even their staff. The pharmacy team should know what is to be done when there is a suspected case of COVID-19 infection. They should also have information on the measures of prevention, including details about disinfectants and proper hand and face hygiene (Facial T-Zone hygiene).

In absence of specific guidelines regarding the measures to be adopted for social distancing (in fact, physical distancing) by the community pharmacies, the approaches enlisted below can be and many of these are being followed by the pharmacies:

- Placing physical barriers such as tables or chairs in the doorways or in front of the pharmacy counter to prevent patients from getting close.

- Dispensing medicines through a small window on the façade or door, like those usually used for night services.

- Putting plastic shield in front of the dispensing area.

- Putting some colorful markings or making boundaries with colored tape on the ground to designate the areas of the pharmacy where patients can stand for ensuring safe distance.

- Restricting the number of people who can be present in the pharmacy at any one time e.g. two in, two out. Another way is by locking the door for some time and then opening to limit the number of people entering the pharmacy.

- If you have a big pharmacy where the products are displayed at the patient/customer's disposal, then the access to these 'products on self-selection' should be restricted to avoid many people touching these products. 


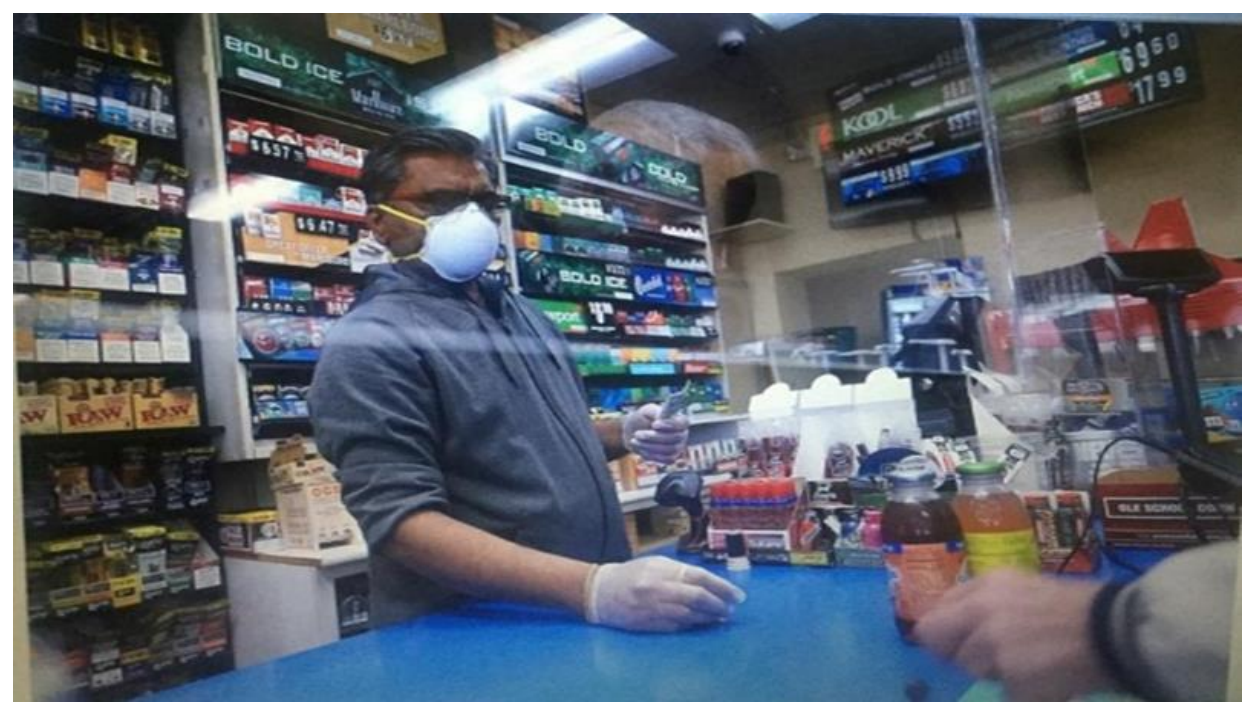

Figure 1 Dispensing of medicines through a small window

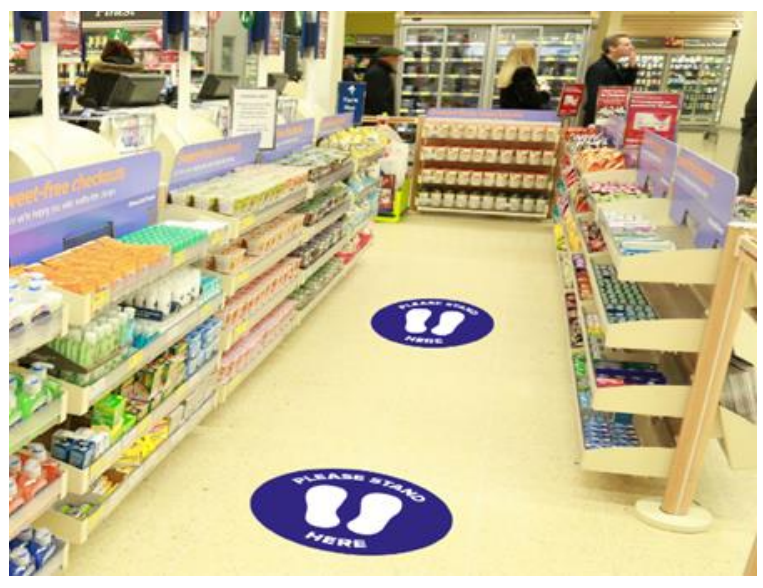

Figure 2 Markings on the floor for safe-distancing

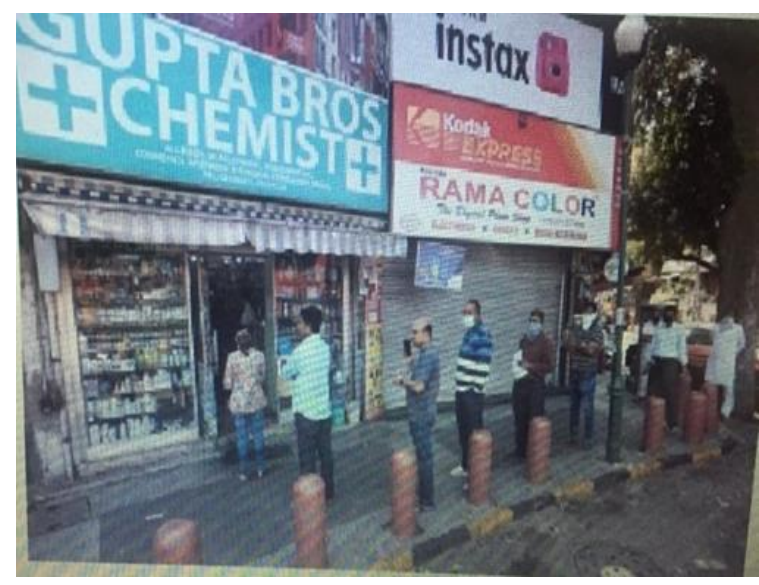

Figure 3 People following safe distancing

In the countries where the impact of COVID-19 is very high, the pharmacies are advised on how to deal with the people presenting with the symptoms at the pharmacy. Such people with 'new continuous cough' and/or fever need to be advised to 'Go home and immediately self-isolate'. They need to contact the government health authorities and get themselves tested. These measures may seem extreme, but have to be adopted in the pharmacies to help prevent the spread of COVID-19 between patients and to the staff, especially to those who may be vulnerable to infection or in highrisk groups.

\subsubsection{Reducing interactions}

It is also important for the staff to reduce interaction with the patients. Despite all the precautions taken, there will be some interaction with the patients, may be while handing over their medicines to them or while they make payment for the medicines. In order to comply with the ever-important hand-hygiene and to reduce the spread at this point, measures such as encouraging patients to pay using contactless payment when possible, or placing money in baskets/bowls, may further reduce the need for physical contact. The staff have to be encouraged to thorough hand washing as per the WHO's current COVID-19 guidelines [6]. 


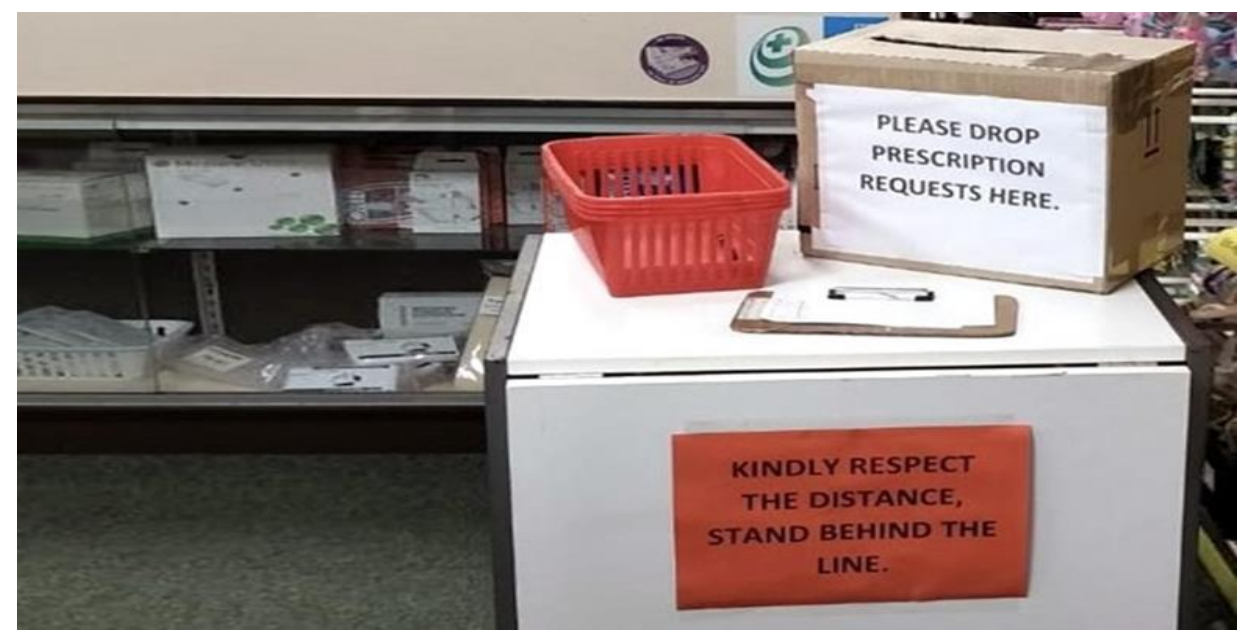

Figure 4 Encouraging patients to place money in basket and prescription in box

One can also adopt to a practice where the patients call to ask for items and then collect them, limiting patient interaction and time period of their presence in the pharmacy.

During the pandemic, the pharmacies should prioritize the dispensing of only medicines and essential medical devices over the non-essential commodities.

\subsubsection{Using Administrative controls}

In the times of pandemic, the primary functions of the pharmacy is dispensing medicines and medicine delivery. This should be clearly communicated to the public. The secondary function of retailing other commodities should be completely stopped. A sign/instruction stating the sale of only medicines, which the customers can read should be placed on the door. This helps to limit the entries in the store at any given time. A poster can also be placed in the store window indicating that patients may have to wait for a longer time [7]. The pharmacy can direct the patients to call the pharmacy with queries rather than turning up in person.

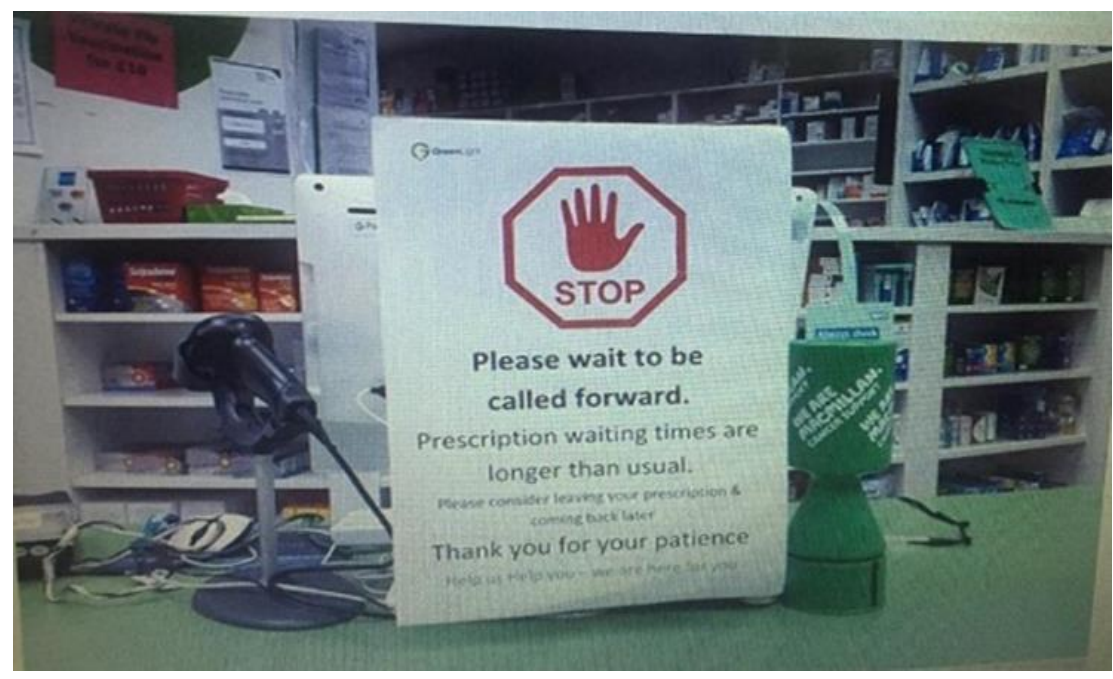

Figure 5 Poster indicating longer waiting time

Posters/instruction stating when it is suitable for individuals to visit the pharmacy can be displayed. The instructions may also be conveyed by placing a leaflet along with the patient's medicine which is being delivered.

In addition, the pharmacies can also display other helpful information such as the latest advice/s on COVID-19 from the government such as: 
- Whom to contact if they are concerned about their symptoms (e.g. calling the helpline number of the government health regulatory).

- If you are self-isolating, have fever, cough, or shortness of breath, please do not come in to the pharmacy. Go home.

- If you need medicines, ask friends/family to come in for you.

- For advice about coronavirus, visit the website of National Centre for Disease Control.

As the global COVID-19 scenario and status is changing constantly, being evident from the fact that the statistical figures about the morbidity and mortality become outdated every hour; this information about the advice/guidelines should be reviewed daily to ensure it is up to date and should be changed in accordance with the government guidelines.

At times, it is likely that the employees receive abuse from some patients and public, due to longer waiting period or many a times on non-receipt of some medicine due to medicine shortages the pharmacies are facing today. Although most of this may be due to panic, anxiety and apprehension, but is absolutely not acceptable.

The following may be considered an abuse:

- Using inappropriate language with the staff;

- Any physical violence towards any of the staff or other patients, like pushing etc.

- One must adopt 'zero tolerance policy' for such abuse. You can refuse to serve to such patients and they have to be politely instructed to leave the place. Pharmacies which do not have their own security support, can definitely call police.

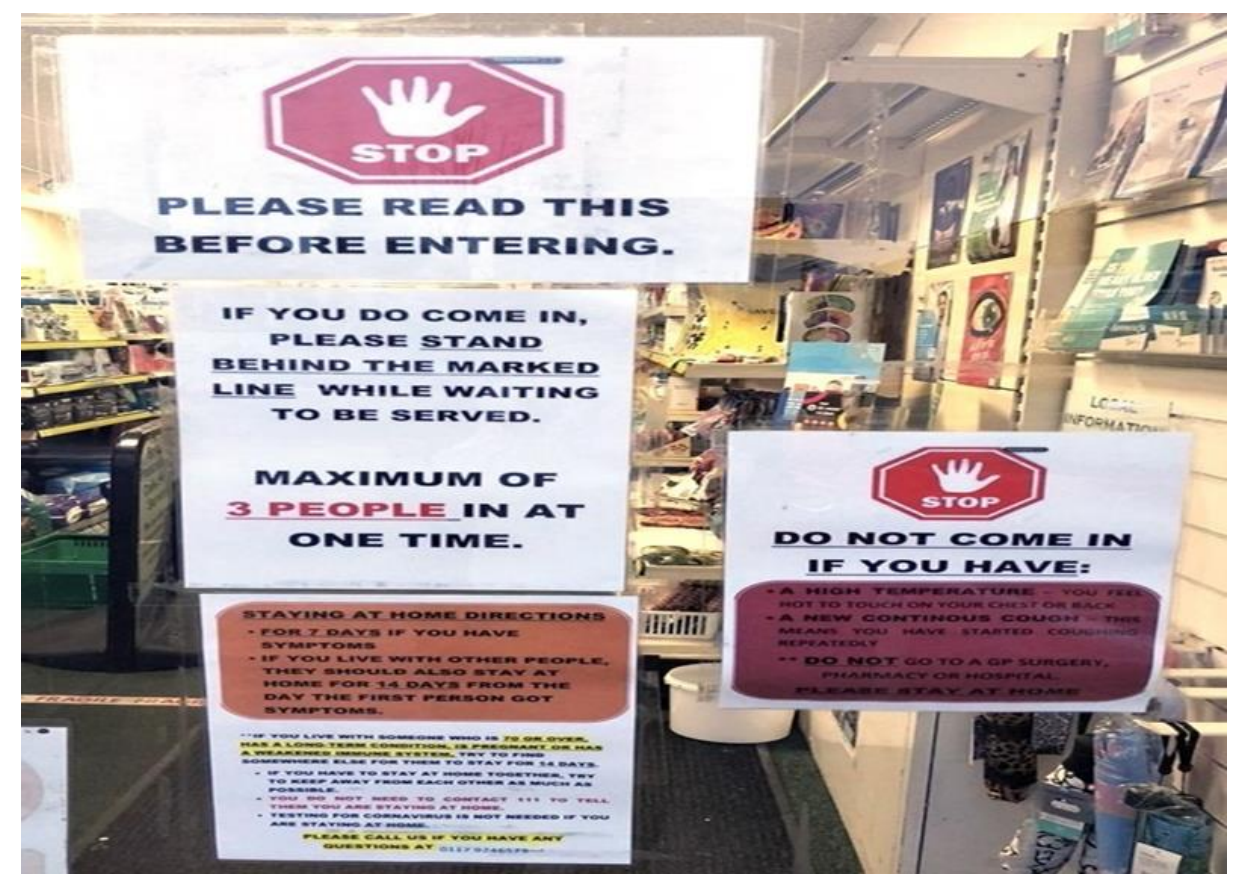

Figure 6 Advisory for patients

\subsection{Caring for Staff safety}

It is of utmost importance to keep your staff protected and fit in the situation of pandemic. It is evident that the pharmacy staff has to be on forefront in the coming time, may be for coming weeks or months. It is necessary to be honest with the staff regarding the current situation, at the same time advise the staff on all possible precautionary measures, as obviously the staff is put at a higher risk due to their patient-facing role.

Here it is advisable to put the staff duty 'On Rota'. This means adjusting the duties of the staff in such a way that everyone gets an opportunity to remain away from being patient facing.

Avoid using the services of the vulnerable staff [8], if any, who is at a higher risk with respect to COVID-19, as those with pregnancy or other morbidities such as diabetes, chronic cardiovascular or respiratory conditions etc. 
However, if at all you have to avail their service, ensure that they have either no or very little direct contact with the patients. In fact, such staff may be used for the work that does not require patient or colleague contact such as being on phone lines and answering queries. It is also to be ensured that none of the staff is showing any symptoms and that there is no one in their household with COVID-19 symptoms, and if so, they need to self-isolate for 14 days.

For reducing the risk of transmission - for staff, following measures seem to be imperative:

- Hand-hygiene: Train staff on proper washing of hands and make sure the staff regularly wash their hands after handling the prescription, dispensing medicines or touching the fomites which could have been contaminated and also after interacting with the patients.

- It is the best practice that the staff wears the uniform which should ideally be short-sleeved or rolled up sleeves.

- All pharmacy staff at the counter should be encouraged to wear a face mask to protect themselves and the public. Whenever you wear a mask, hygiene and disinfection of the hands and face should be performed before and after.

- Keep only the essential objects at the counter.

- Remove wristwatches or jewelry when in the pharmacy - this not only helps prevent the transmission of COVID-19, but will also help with hand washing.

- Keep an alcohol-based sanitizer handy to disinfect the hands after attending to each patient/customer.

- Regularly clean and disinfect the surfaces, including counters, chairs, door handles, stationery, phones, keyboards, mouse etc. Keep the equipment/devices which are difficult to clean such as keyboards covered with transparent bags.

- Encourage contactless payments and if mandatory, wash hands after each transaction and after counting cash.

- Point-of-care tests such as measurement of blood pressure, blood sugar etc. or any other service, if authorized, should be attempted in full compliance with the protective guidelines like use of masks, gloves etc. However, such services should be totally interrupted if the patient shows any symptom of respiratory infection.

\subsection{Adequate stocking of medicines}

In the pandemic, just like other countries, Indian Pharma sector also seems to be worried about the consistent supply of pharma products due to possible disrupted supply of the active pharmaceutical ingredient (API) and the intermediates, as approximately 70\% of India's total API requirement is met by imports from China [9], the country first hit by COVID-19.

In order to assess the impact of the lockdown over manufacturing and supply chain of medicines, the DCGI (The Drug Controller General of India) has asked the State drug controllers to provide details regarding the no. of manufacturing units operational at present, \% of average daily production of formulations and of the APIs and the \% of employees as compared to Pre-COVID situation.

There was an anticipation regarding the supply of medicines in these times as the demand for medicines rises in the pandemic. However, the government is making all attempts to put the processes in place to reduce the impact of shortage of medicines and to be able to provide a consistent supply of critical products from its stores.

In the current situation, there is a likelihood of an unprecedented demand for OTC painkillers and antipyretics as the pandemic spreads [10].

The pharmacies must guarantee the adequate supply of the medicines, particularly antiviral drugs, antimicrobial agents as also the drugs for supportive treatment such as analgesics, antipyretics etc.

There is a sudden additional demand for the medical devices such as thermometers, face-masks, gloves, goggles and some other items such as soaps, hand wash, disinfectants, tissue paper etc.

It is also possible that the pharmacies do not receive delivery of entire amount as ordered, the main reason being they are ordering a lot more than they would normally do, so not getting the whole delivery the first time. Here it is strategic to prioritize what is the requirement of your local community. 
If your pharmacy is also selling non-medicine products, it is important to realize that at present, there is a surge in the demand for health-boosting products such as Oats, health drinks, milk powder, some ayurvedic immunity boosters such as Chyavanprash, and even Vitamin $\mathrm{C}$ tablets.

It is also apt to consider limiting the quantity of items that can be sold from the pharmacy. However, this should be taken on a case-by-case basis, for example, one individual could be coming to collect medicine for elderly relatives who are unable to leave the house and so it may be acceptable to provide multiple items to one individual for this reason. For prescription items, if you are short of an item, it is practical to inform the general practitioner/physician about the shortages so that they can prescribe the alternatives or you can contact another local pharmacy that may be able to provide the patient with the item.

It is logical here to discuss about Repeat Prescription or Post-dated prescriptions. The first thing to do is to politely request people not to ask for medicines unless they really need it, because the consequent shortage may put somebody else 'at risk'. Never-the-less, looking at the potential effect of the COVID-19 pandemic, it would be in the patient's best interest for medicines already dispensed to be supplied from the pharmacy rather than not supplied at all. However, it is necessary to counsel the patients to only purchase medicines according to the need and avoid hoarding.

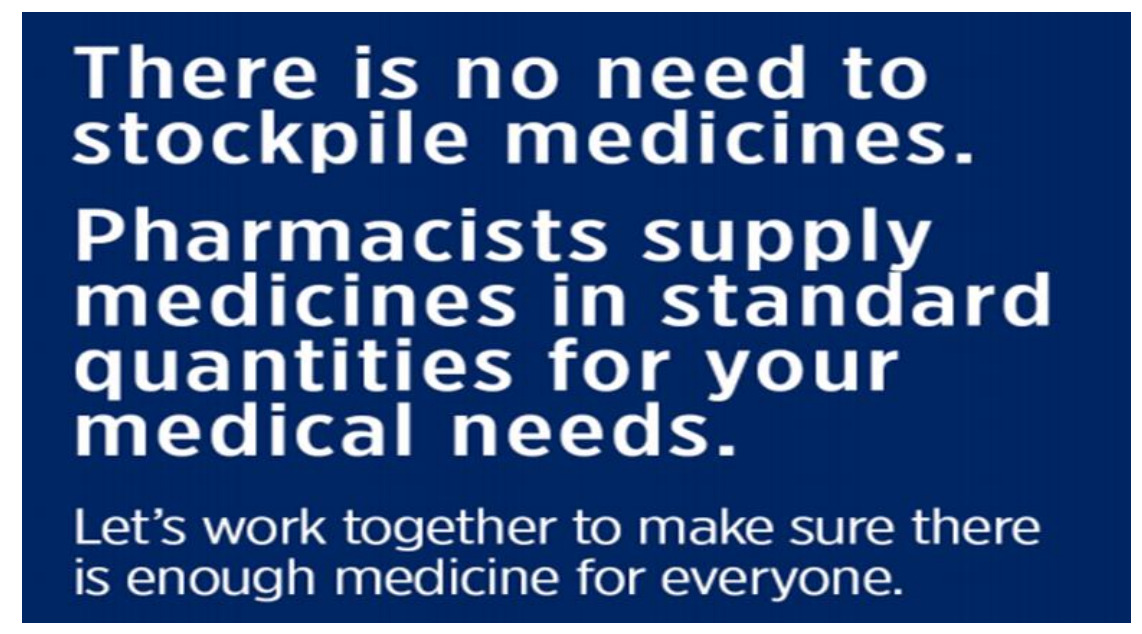

Figure 7 Poster urging patients against medicine hoarding

Further, whenever possible, medicine supplies from the distributor should be taken by the pharmacy staff avoiding anyone external to enter the pharmacy. The cases/boxes used by wholesale distributors for the medicine delivery must be cleaned and disinfected before taking inside the pharmacy.

\subsection{Efficient and safe deliveries of medicines}

In the pandemic, you may have higher number of medicines that have to be delivered to the patients. To ensure the proper supply of medicines to the patients and the community, the pharmacies may have to provide the home delivery of medicines. In fact the pharmacies that are able to provide this service are encouraged to do that, as it is very important for the patients who are at higher risk (age over 70 years or below 70 but with comorbidities or have reduced mobility).

In India, on 26th March, 2020, the Ministry of Health, issued a notification allowing a pharmacy retailer for doorstep delivery of essential medicines, including those under Schedule H, but subject to following conditions [11]:

- Sale of a drug that falls under Schedule H of the Drugs and Cosmetics Act shall be based on receipt of prescription physically or through email.

- The licensee shall submit an e-mail ID for registration with the licensing authority if prescriptions are to be received through e-mail for these drugs.

- The drug delivery can only be done within the same district.

- The prescription shall be dispensed only if it is presented to the licensee within 30 days in case of chronic diseases whereas in case of acute cases, the prescription shall be dispensed only if it is presented to the licensee within seven days of its issue. 
- The bill or cash memo shall be sent by the return e-mail and records of all such transactions shall be maintained by the licensee.

- Home delivery of medicines was not permitted for medicines in Sch H1, or Sch X, or controlled substances.

- The above order was taken out by the government as a part of the strategy to keep people at home, and reach the medicines to those people who cannot come out.

- However, since many doctors are not practicing, patients would have difficulty getting prescriptions for their chronic conditions, if their earlier prescriptions are already beyond 30 days.

The legal sanctity of the order allowed the drug regulators of various states to encourage the pharmacies to do home deliveries in this hour of crises, and regulators worked with the chemists associations in various regions for giving publicity to this activity, so that the public do not panic and can receive their medicines without coming out.

Following may be considered to protect the person in charge of the actual delivery and others when delivering to people including to those in isolation:

- Avoid any direct contact with the patient and their personal objects. If you leave the medicines outside the door of the patient/customer or in some other designated place as per telephonic instruction from the patient, you should make sure you wait for the person to open the door and receive the medicines while keeping a safe distance.

- Avoid touching surfaces that could be contaminated like doorbells, gates, grills etc., preferably use gloves or later sanitize if hand washing is not possible.

- Avoid touching your mouth, nose, or eyes.

- Keeping a safe distance of at least 1 meter away from people.

- Plan routes so those in self-isolation receive deliveries at the end

- Washing your hands or using sanitizer regularly between each drop off and regularly in between such as after eating, after breaks, before starting and finishing work.

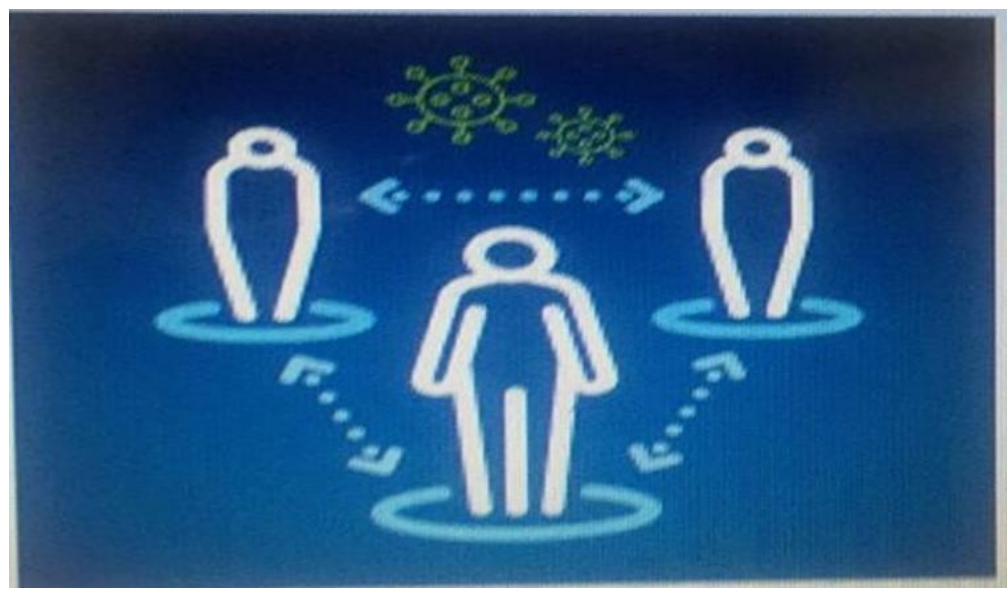

Figure 8 Safe distancing

\subsection{Maintaining the hygiene and control measures for infection}

\subsubsection{Cleaning the pharmacy}

Although it is important to clean the pharmacy on a regular basis, this should now be done in a meticulous manner and much more regularly. There are some areas which if contaminated would dangerously increase the risk and include the light switches, refrigerator doors, door handles, soap dispensers, table and counter tops, chairs, computer key board etc. These areas have to be cleaned and disinfected with more attention.

It is also advisable to get in touch with some such service provider companies and discuss with them the cleaning needs for your premises. Periodic deep cleaning and disinfection of the area would be logical.

While completing the general cleaning of the pharmacy [12], you should: 
- Use disposable cloths, paper roll or mop heads when possible

- Clean and disinfect all hard surfaces, floors, chairs and door handles

- Use detergent disinfectant

- Regularly wipe down 'high-touch' areas (e.g. light switches, refrigerator doors, drawer pulls, sink handles, soap dispensers).

Wearing gloves would be appropriate as the substance you use to clean may be harmful. These gloves should then be washed if you are going to re-use them.

\subsubsection{Waste management}

The store should have enough waste bins. Precautions such as double-bagging used tissues or disposable cleaning cloths and separating them from other waste for 72 hours before placing in external waste bins may be necessary [13].

\subsection{Complying with various restrictive measures on the sale of some medicines and additional duty of informing the authorities}

During the pandemic, there are guidelines by the District Collectors, the police and the drug administration (and later Orders in certain areas high-hit by COVID-19) to the retail pharmacies in their jurisdiction to dispense medicines for cough, cold and fever with stringency and only against the prescription of a doctor. Pharmacies are asked to keep detailed records of the medicines sold and send a daily report of the same along with the details of the patient and of the doctor who prescribed the medicine to the local drug regulator and the police on Email/ WhatsApp.

The pharmacies were also asked to take down and send the details of any patient who comes to the pharmacy asking for medicines for cough, cold or fever, or who shows any signs or symptoms of these - to the police, and FDA, and also direct the patient to visit the nearest flu clinics/government clinic/hospitals.

The reason behind this was that and due to the access to some fake news on the social media platforms, people are hesitant to self-report of COVID-19 symptoms, and are not going to hospital/ clinics. It is anticipated that they could try to self-medicate. These were stringent measures put in place by the various administrative heads to trace out as many suspects of COVID-19 as possible to stop the transmission.

In addition, various Guidelines/directives were issued in various locations/hotspots, in specific areas where there was strict curfews, to restrict the moving out of people from their homes. The administration restricted/reduced the timings of opening of pharmacies, so that with shortened duration, there would be lesser movement of people and reduce the risk of spread.

\section{Conclusion}

It is obviously seen that in this pandemic, out of necessity, the community pharmacists have created a work atmosphere and an environment which is now conciliated to infection control and is ready to face such future disaster in a better way.

However, despite of their services in health care sector, in India, the community pharmacists have not been able to create/establish a very impressive image before the health regulatory authorities or even the public, and they must put in all efforts to change their image of being into a 'profession' and not a 'trade'.

\section{Compliance with ethical standards}

\section{Acknowledgments}

All the authors are grateful to the management of their respective institutes for their encouragement for preparation of this article.

\section{Disclosure of conflict of interest}

There is no conflict of interest regarding the publication of this article. 


\section{References}

[1] FIP (International Pharmaceutical Federation). (2020). Health Advisory on "COVID-19: Guidelines for Pharmacists and the Pharmacy Workforce".

[2] Gov. uk. PM statement on coronavirus. (2020).

[3] Andaolo D. (2020). 'Unprecedented demand' for OTC painkillers as COVID-19 outbreak spreads. Pharm J.

[4] Ministry of Health and Family Welfare. (2020).Social Distancing advisory.

[5] Interim Recommendations by the World Health Organization on obligatory hand hygiene against transmission of COVID-19. (2020).

[6] Royal Pharmaceutical Society. (2020).Coronavirus (COVID-19) Pharmacist updates and information.

[7] National Centre for Disease Control "Guidelines for workplace of COVID-19 case", 1727004/2020/0/0 IDSPNCDC. (2020).

[8] The Pharmaceutical Journal by Debbie Andals. (2020).

[9] Notification by the Ministry of Health, issued on 26th March, 2020 entitled "COVID-19 lockdown: Health Ministry allows doorstep delivery of essential medicines"

[10] Ministry of Health and Family Welfare. (2020). Guidelines on disinfection of common public places including offices.

[11] COVID-19: guidance for supported living provision. (2020).

\section{How to cite this article}

Desai DM, Patil RB and Desai YM. (2020). Community pharmacists: Frontline COVID-19 warriors and the approaches for the safe management of the pharmacies during the pandemic. World Journal of Advanced Research and Reviews, 6(2), 176-185. 